\title{
Wideband Shaped-Beam Reflectarray Design Using Support Vector Regression Analysis
}

\author{
Daniel R. Prado, Jesús A. López-Fernández, Manuel Arrebola, Senior Member, IEEE, \\ Marcos R. Pino and George Goussetis, Senior Member, IEEE
}

\begin{abstract}
In recent years, machine learning techniques (MLTs) have been applied to accelerate analysis and design of electromagnetic devices. Algorithms such as artificial neural networks or support vector machines for regression (SVRs) have been proposed for the design of large reflectarrays for space applications at a single frequency. However, multi-frequency optimization of such large antennas has not been tackled with MLTs. In this letter and for the first time, we propose a technique based on the use of SVR analysis to obtain the reflection coefficients to accelerate the design of a very large shaped-beam reflectarray for direct broadcast satellite in a $15 \%$ bandwidth. An in-house method of moments based on local periodicity is employed to generate samples to train the SVRs for each considered frequency. Then, the surrogate model is used for a design at central frequency, which is used as starting point for a wideband design procedure that is accelerated more than an order of magnitude without a significant loss of accuracy. It is shown that, by virtue of the proposed methodology, the minimum copolar gain in the coverage zone is improved more than $10 \mathrm{~dB}$ at the upper frequency while maintaining a computationally efficient design procedure.
\end{abstract}

Index Terms-Machine learning, support vector regression (SVR), wideband reflectarray antenna, shaped-beam, direct broadcast satellite, generalized intersection approach

\section{INTRODUCTION}

$\mathbf{T}$ HE main drawback of printed reflectarrays is their inherent narrow bandwidth, that is primarily attributed to two factors: the poor bandwidth of narrowband resonant elements, which is usually around $3 \%-5 \%$, and the differential spatial phase delay [1], [2]. The first problem may be solved by employing wideband printed elements which introduce several resonances [3]-[5]. Also, the use of sub-wavelength elements may improve the bandwidth [6], although at the expense of reducing the phase-shift range [7], limiting the design of

This work was supported in part by the Ministerio de Ciencia, Innovación y Universidades under project TEC2017-86619-R (ARTEINE); by the Ministerio de Economía, Industria y Competitividad under project TEC2016-75103C2-1-R (MYRADA); by the Gobierno del Principado de Asturias/FEDER under Project GRUPIN-IDI/2018/000191; by the Gobierno del Principado de Asturias through Programa "Clarín" de Ayudas Postdoctorales / Marie CurieCofund under project ACA17-09; by Ministerio de Educación, Cultura y Deporte / Programa de Movilidad "Salvador de Madariaga" (Ref. PRX18/00424).

D. R. Prado and G. Goussetis are with the Institute of Sensors, Signals and Systems, School of Engineering and Physical Sciences, Heriot-Watt University, Edinburgh, U.K. (email: dr38@hw.ac.uk; g.goussetis@hw.ac.uk).

J. A. López-Fernández and M. Arrebola and M. R. Pino are with the Department of Electrical Engineering, Group of Signal Theory and Communications, Universidad de Oviedo, Gijón, Spain (e-mail: jelofer@uniovi.es; arrebola@uniovi.es; mpino@uniovi.es).

Color versions of one or more of the figures in this paper are available online at http://ieeexplore.ieee.org.

Digital Object Identifier XX.XXXX/LAWP.XXXX.XXXXXXX shaped-beam reflectarrays. More complicated elements may provide at least a $360^{\circ}$ phase-shift [8], although the analysis becomes slower due to the complexity of the unit cell. The second factor may be overcome by adjusting the geometry of the unit cell at several frequencies [3], [9], using true time delay reflectarray elements [10], increasing the $f / D$ ratio [2] or using curved [11], [12] or faceted [13]-[15] reflectarrays.

Machine learning techniques [16] such as neural networks (NNs) have been employed for the analysis [17], [18] and design [19], [20] of reflectarrays. Deep learning NNs were employed in [21] to predict the phase-shift. In [22], kriging was proposed to predict the electromagnetic response of reflectarray elements. Support vector machines (SVMs) for regression (SVRs) were also used to accelerate the analysis of reflectarrays [23], and for the direct optimization of reflectarrays [24], although only at a single frequency, yielding a narrowband design. Another approach to accelerate computations is to employ a Floquet modal expansion with equivalent circuits [25].

In this letter and for the first time, a wideband reflectarray design technique based on the use of SVR analysis to greatly accelerate computations without a significant loss of precision is proposed, obtaining a wideband, dual-linear polarized, shaped-beam reflectarray for direct broadcast satellite (DBS) application. The unit cell is characterized using an in-house method of moments based on local periodicity (MoM-LP), which is employed to generate samples of the electromagnetic behaviour of the unit cell to train the SVR, as well as to validate the final solution. The SVR kernel is Gaussian and the selection of the kernel $(\gamma)$ and soft margin $(C)$ parameters is based on an efficient grid search which greatly accelerates the training process with regard to an exhaustive one. The obtained surrogate model is compared with simulations from MoM-LP, showing a high degree of accuracy. A wideband design based on those surrogate models is then carried out. The final layout almost fulfils requirements in a $15 \%$ bandwidth, demonstrating the capabilities of the SVR-based proposed technique.

\section{SVR Model of the Reflectarkay Unit Cell}

For each frequency, the feed generates an incident field $\left(\vec{E}_{\text {inc }}(f)\right)$ on the reflectarray surface that varies with frequency. Then, the reflected tangential field $\left(\vec{E}_{\text {ref }}(f)\right)$ is related to the incident field through the matrix of reflection coefficients for a given unit cell:

$$
\boldsymbol{R}(f)=\left(\begin{array}{cc}
\rho_{x x}(f) & \rho_{x y}(f) \\
\rho_{y x}(f) & \rho_{y y}(f)
\end{array}\right)
$$




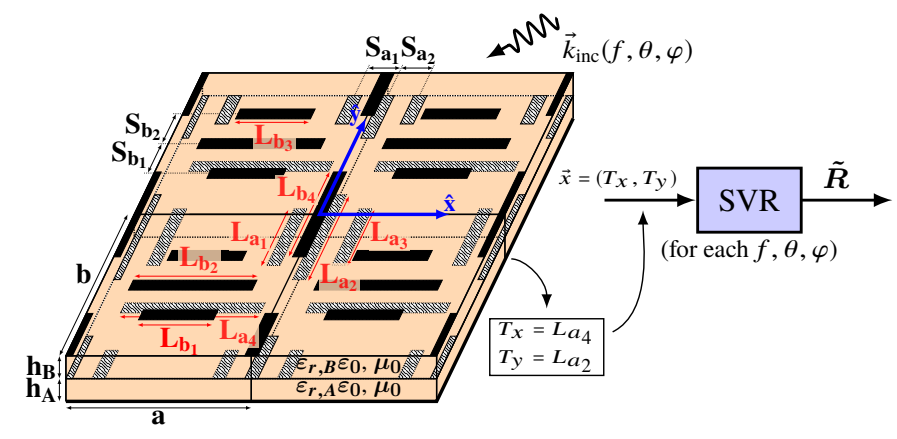

Fig. 1. Sketch of the reflectarray unit cell based on two sets of parallel dipoles for dual-linear polarization and its relation with the SVR of the reflection coefficient matrix. Optimized parameters are shown in red.

This matrix is computed using a full-wave analysis tool, in the present case the MoM-LP described in [26], which analyses the unit cell shown in Fig. 1. The coefficients are complex numbers. $\rho_{x x}$ and $\rho_{y y}$ are known as the direct coefficients and mainly control the copolar pattern for each linear polarization (X and Y, respectively). $\rho_{x y}$ and $\rho_{y x}$ are the cross-coefficients and mainly contribute to the cross-polar pattern. Thus, when performing copolar only synthesis, assuming $\rho_{x y}=\rho_{y x}=0$ is a good approximation [27]-[29]. Once the reflected tangential field is obtained, the far field may be readily computed [1].

The chosen unit cell is shown in Fig. 1. It has been designed to provide broadband performance in dual-linear polarization [30], and it is comprised of two sets of four parallel dipoles. Each set controls the phase-shift for a linear polarization by adjusting the lengths of the dipoles. Thus, the width of the dipoles will be fixed to $0.5 \mathrm{~mm}$ and the separation between adjacent dipoles to $4 \mathrm{~mm}$. Commercial substrates were chosen, the Arlon AD255C for layer A with $h_{A}=2.363 \mathrm{~mm}$ and $\varepsilon_{r, A}=2.17-j 0.0020$, and the Diclad 880 for layer B, with $h_{B}=1.524 \mathrm{~mm}, \varepsilon_{r, B}=2.55-j 0.0036$ [31].

The goal of the SVR [32] is to obtain surrogate models of (1) for each cell and frequency. We consider two input variables for each SVR, $T_{x}$ and $T_{y}$, related to the dipole lengths as [31]:

$$
\begin{aligned}
& L_{a_{4}}=T_{x} ; \quad L_{b_{1}}=L_{b_{3}}=0.63 T_{x} ; \quad L_{b_{2}}=0.93 T_{x} \\
& L_{b_{4}}=0.95 T_{y} ; \quad L_{a_{1}}=L_{a_{3}}=0.58 T_{y} ; \quad L_{a_{2}}=T_{y} .
\end{aligned}
$$

$T_{x}$ and $T_{y}$ allow to control the phase-shift for linear polarizations $\mathrm{X}$ and $\mathrm{Y}$, respectively. In addition, $T_{x}, T_{y} \in[4,10] \mathrm{mm}$ to avoid highly resonant behaviour of the unit cell.

We estimate the phase of the direct reflection coefficients by modelling their real and imaginary parts. In addition, we separately model their magnitude in order to increase the accuracy [23]. Thus we model $N_{c}=6$ real-valued functions per unit cell. Let $\rho_{R, I}$ be the real or imaginary part of any reflection coefficient in (1). Then, the SVR provides an estimation of $\rho_{R, I}$, noted as $\tilde{\rho}_{R, I}$ :

$$
\tilde{\rho}_{R, I}(\vec{x})=\sum_{k=1}^{N_{s}}\left[\left(\alpha_{k}^{-}-\alpha_{k}^{+}\right) K\left(\vec{x}_{k}, \vec{x}\right)\right]+b,
$$

where $\vec{x}=\left[T_{x}, T_{y}\right]$ is a vector with the geometrical features of the unit cell used as input variables for the SVR; $\vec{x}_{k}$ is
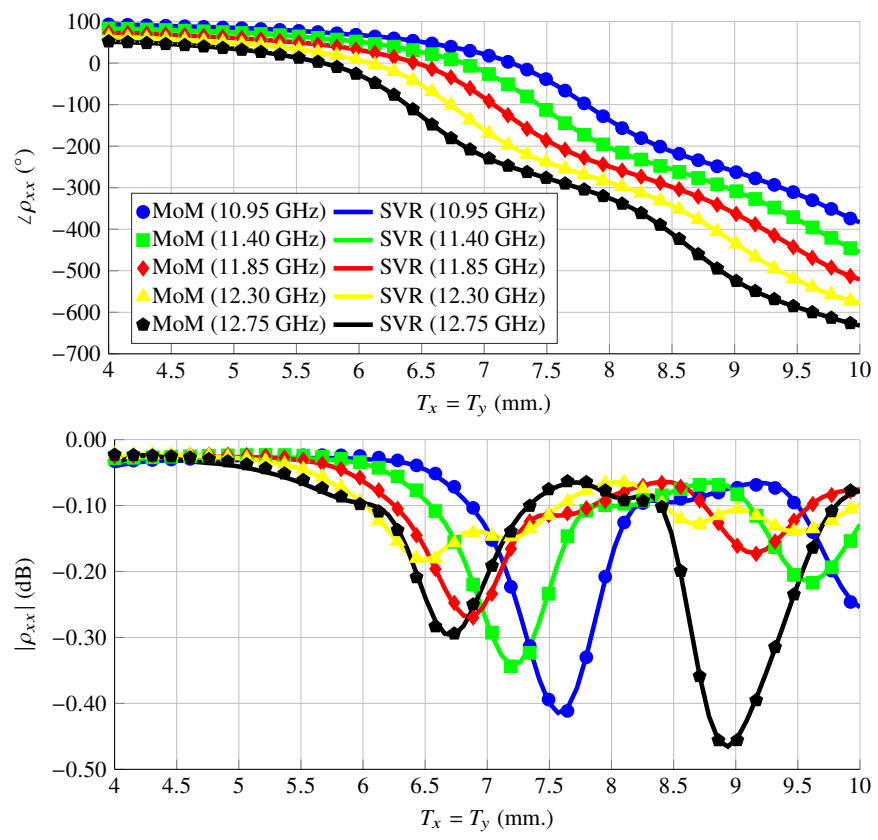

Fig. 2. For the direct reflection coefficient $\rho_{x x}$ with an oblique angle of incidence $(\theta, \varphi)=\left(30^{\circ}, 50^{\circ}\right)$, comparison at five different frequencies between MoM-LP simulations and the SVR surrogate model for phases (top) and magnitudes (bottom).

the $k$-th support vector; $N_{s}$ is the number of support vectors; $\alpha_{k}^{-}$and $\alpha_{k}^{+}$are the $k$-th optimal Langrange multipliers; $b$ is the offset; and $K$ is the kernel function, which in this case is a Gaussian bell $K\left(\vec{x}_{k}, \vec{x}\right)=\exp \left(-\gamma\left\|\vec{x}_{k}-\vec{x}\right\|^{2}\right)$, where $\gamma$ is a tunable parameter. Eq. (3) minimizes a regularized risk functional [33] that takes into account the flatness of $\tilde{\rho}$ and the empirical errors (scaled by parameter $C$ ).

In addition, we consider a discrete set of $N_{a}=52$ angles of incidence to the reflectarray cells given by: $(\theta, \varphi) \in$ $\left\{5 \times \varphi_{8}, 15 \times \varphi_{7}, 25 \times \varphi_{5}, 30 \times \varphi_{2}\right\}$ where $\varphi_{N}= \pm\{10+$ $20 n, n=0,1, \ldots, N\}$ and $\times$ stands for the Cartesian product. This set is reduced to $N_{a}=26$ using symmetries. Notice that, due to the reflectarray optics, high values of $\theta$ are associated with low ranges in $\varphi$. Finally, we also model separately each considered frequency $(10.95 \mathrm{GHz}, 11.40 \mathrm{GHz}$, $11.85 \mathrm{GHz}, 12.30 \mathrm{GHz}$ and $12.75 \mathrm{GHz}$ ), which yields $N_{f}=5$. Thus, a total of $N_{c} N_{a} N_{f}=780$ models are generated.

Obtaining each surrogate model involves the training of a SVR, and its statistical effectiveness depends on the proper selection of the set $(C, \gamma)$. A total of 2500 samples in a random grid for each model are generated. We use cross-validation to select the optimal $(C, \gamma)$ following [23], with $70 \%$ of the samples for training, $15 \%$ for validation and $15 \%$ for test. The chosen number of training samples is a conservative trade-off between accuracy and training time for this kind of problems [23]. A mean training time of $38 \mathrm{~s}$ is achieved in an Intel Core i7-5600U at $2.6 \mathrm{GHz}$. The mean test error for all coefficients, all angles of incidence and frequencies has been calculated according to [23, Eq. 11] and is $-40.9 \mathrm{~dB}$. This ensures a high degree of accuracy in the prediction of the direct reflection coefficients. Fig. 2 shows the phase and magnitude of $\rho_{x x}$ for oblique incidence at the five frequencies. It is shown that the 
Table I

MINIMUM COPOLAR GAIN FOR BOTH LINEAR POLARIZATIONS (X AND Y) AT THE FIVE FREQUENCIES OF INTEREST COMPARING SIMULATIONS WITH THE MOM-LP TOOL (USING THE REAL ANGLES OF INCIDENCE AT EACH REFLECTARRAY ELEMENT AND THE SAME ANGLES USED BY THE SVR) AND SVR.

\begin{tabular}{|c|c|c|c|c|c|c|c|c|c|c|c|}
\hline & \multirow[t]{2}{*}{ Tool } & \multicolumn{2}{|c|}{$10.95 \mathrm{GHz}$} & \multicolumn{2}{|c|}{$11.40 \mathrm{GHz}$} & \multicolumn{2}{|c|}{ 11.85 GHz } & \multicolumn{2}{|c|}{$12.30 \mathrm{GHz}$} & \multicolumn{2}{|c|}{$12.75 \mathrm{GHz}$} \\
\hline & & $\mathrm{X}$ & $\mathrm{Y}$ & $\mathrm{X}$ & $\mathrm{Y}$ & $\mathrm{X}$ & $\mathrm{Y}$ & $\mathrm{X}$ & $\mathrm{Y}$ & $\mathrm{X}$ & $\mathrm{Y}$ \\
\hline \multirow{2}{*}{ Initial (in dBi) } & MoM-LP (real ang. inc.) & 25.99 & 25.94 & 28.79 & 28.54 & 30.11 & 30.06 & 26.03 & 28.21 & 15.15 & 23.69 \\
\hline & SVR & 26.08 & 25.96 & 28.75 & 28.56 & 29.90 & 30.10 & 26.38 & 28.22 & 19.93 & 23.85 \\
\hline \multirow{2}{*}{ Optimized (in dBi) } & MoM-LP (real ang. inc.) & 27.75 & 27.84 & 28.39 & 28.67 & 28.33 & 28.81 & 28.64 & 29.08 & 26.75 & 28.11 \\
\hline & MoM-LP (SVR ang. inc.) & 27.72 & 27.81 & 28.32 & 28.65 & 28.02 & 28.87 & 28.44 & 29.13 & 27.41 & 28.19 \\
\hline
\end{tabular}

SVRs provide accurate results with regard to the MoM-LP. The mean absolute deviation for all the phase-shift curves in Fig. 2 is $2.25^{\circ}$, while for the magnitude is $-56.8 \mathrm{~dB}$. Similar results were obtained for other coefficients and angles of incidence. Finally, it is worth noting that including the angles of incidence as input variables of the SVR does not increase the regression accuracy, while considerably increasing training time.

\section{BROADBAND REFLECTARRAY DESIGN}

\section{A. Central Frequency Design}

A rectangular printed reflectarray in single-offset configuration is considered [9]. The reflectarray is comprised of $74 \times 70$ elements, with a periodicity of $14 \times 14 \mathrm{~mm}^{2}$. A Gaussian feed horn antenna from Flann Microwave was selected, and it is modelled as a $\cos ^{q} \theta$ function, with the $q$ parameter matching the measured patterns. The feed generates an illumination taper of $-14.8 \mathrm{~dB},-17.0 \mathrm{~dB},-18.5 \mathrm{~dB},-22.3 \mathrm{~dB}$, and $-25.3 \mathrm{~dB}$ at $10.95 \mathrm{GHz}, 11.40 \mathrm{GHz}, 11.85 \mathrm{GHz}, 12.30 \mathrm{GHz}$ and $12.75 \mathrm{GHz}$, respectively. In addition, the feed phase center is placed at $(-358,0,1070) \mathrm{mm}$ with regard to the reflectarray center.

The same European coverage as in [9] is considered, corresponding to a satellite in geostationary orbit at $10^{\circ} \mathrm{E}$ longitude. The goal is to achieve a minimum copolar gain of $28 \mathrm{dBi}$ in a $15 \%$ frequency band $(10.95-12.75 \mathrm{GHz})$.

First, the generalized intersection approach (IA) [34] at central frequency is applied for a phase-only synthesis in several stages, as described in [35]. In this way, the required phase-shift for both linear polarizations is obtained such that the radiated far field fulfils the requirements. Then, by using a zero-finding routine, the values of $T_{x}$ and $T_{y}$ are sought for each reflectarray element to match the required phase-shift at central frequency. The use of the SVR allows to accelerate the analysis and design procedure more than three orders of magnitude. Fig. 3(b) shows the radiation pattern for $\mathrm{Y}$ polarization at central frequency $(11.85 \mathrm{GHz})$. It fully complies with the specifications at that frequency. However, as it can be seen in Figs. 3(a) and 3(c), it is far from complying at extreme frequencies $(10.95 \mathrm{GHz}$ and $12.75 \mathrm{GHz})$.

\section{B. Optimization Algorithm}

For the multi-frequency optimization, the generalized IA is also employed. In particular, the algorithm described in [36] which is adopted for reflectarray antennas. The functional which is minimized in the backward projector now takes into account the far field of the reflectarray at several frequencies:

$$
F=\sum_{f=1}^{N_{f}} \sum_{k=1}^{M}\left\{W_{f}\left(\vec{r}_{k}\right)\left[G_{f}^{\prime}\left(\vec{r}_{k}\right)-G_{f}\left(\vec{r}_{k} ; \tilde{\rho}_{f}\right)\right]\right\}^{2} .
$$

In (4), $M$ is the number of observation points where the far field (gain) is computed; $\vec{r}_{k}=(u, v)_{k}, u=\sin \theta \cos \varphi, v=$ $\sin \theta \sin \varphi ; W_{f}$ is a weighting function which depends on the frequency and observation point; $G_{f}^{\prime}$ is the reference gain; and $G_{f}$ is the current gain radiated by the reflectarray which depends on the SVR model $\left(\tilde{\rho}_{f}\right)$, and by extension, on the optimizing variables $\left(T_{x}\right.$ and $\left.T_{y}\right)$. Eq. (4) represents a weighted Euclidean distance between the current and reference gains [36], and it is minimized by the IA employing the LevenbergMarquardt algorithm [29] in the backward projector.

\section{Results for Wideband Performance}

For the multi-frequency optimization, the design at the central frequency is employed as starting point. The optimization is done in several steps, increasing progressively the number of optimizing variables to improve convergence [34]. This is done by selecting reflectarray elements from the center outwards in concentric circles. In the last step, all elements are optimized at the same time, thus having a total of 10360 optimizing variables $\left(T_{x}\right.$ and $T_{y}$ for each reflectarray element). Fig. 3 shows the initial and optimized radiation patterns for $\mathrm{Y}$ polarization at central $(11.85 \mathrm{GHz})$ and extreme frequencies $(10.95 \mathrm{GHz}$ and $12.75 \mathrm{GHz})$. The layout was simulated with both MoM-LP using the real angle of incidence at each reflectarray element (solid lines) and SVR with the discretized angles (dashed lines). As it can be seen, the SVR-based simulation predicts the radiation pattern with a high degree of accuracy, as it was expected from the results of the reflection coefficients shown in Section II. For these three frequencies, the minimum copolar gain is $27.84 \mathrm{dBi}, 28.81 \mathrm{dBi}$ and $28.11 \mathrm{dBi}$ at $10.95 \mathrm{GHz}, 11.85 \mathrm{GHz}$ and $12.75 \mathrm{GHz}$, respectively. Although at $10.95 \mathrm{GHz}$ it does not achieve a minimum copolar gain of $28 \mathrm{dBi}$, it fulfils specifications in $90.3 \%$ of the coverage surface.

Table I summarizes the results for both linear polarizations at the five frequencies for the initial and optimized layouts. The Table includes simulations of the layouts with MoMLP and SVR to assess the accuracy of the surrogate models. In addition, the MoM-LP simulations were carried out for 


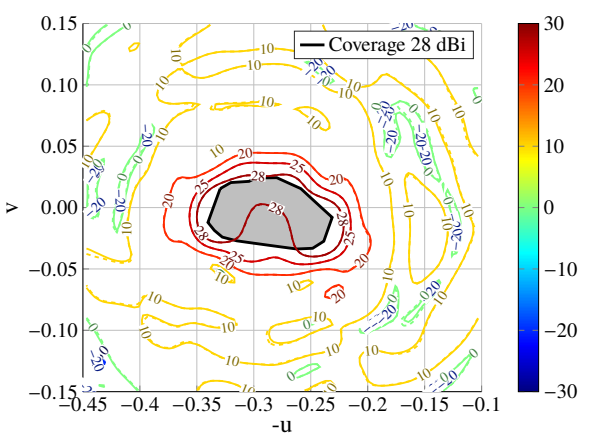

(a)

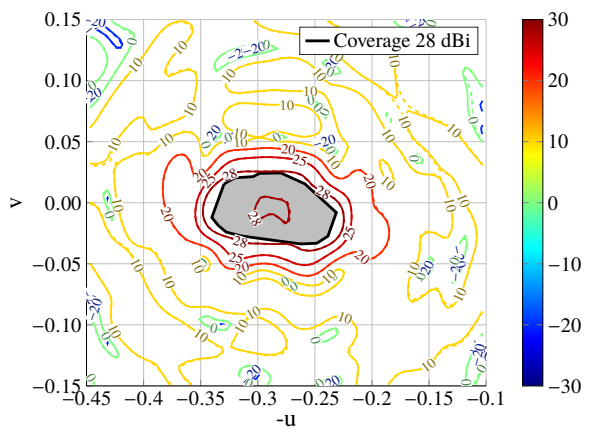

(d)

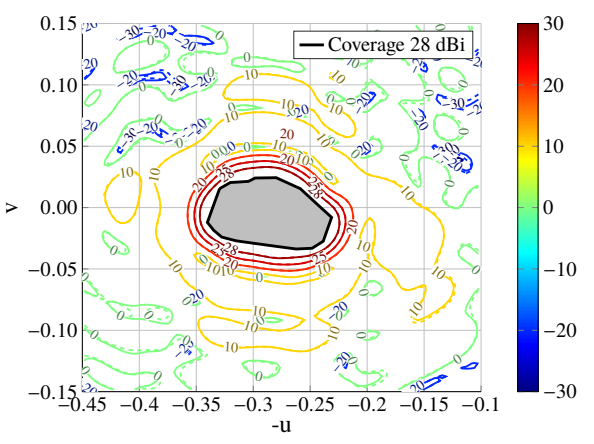

(b)

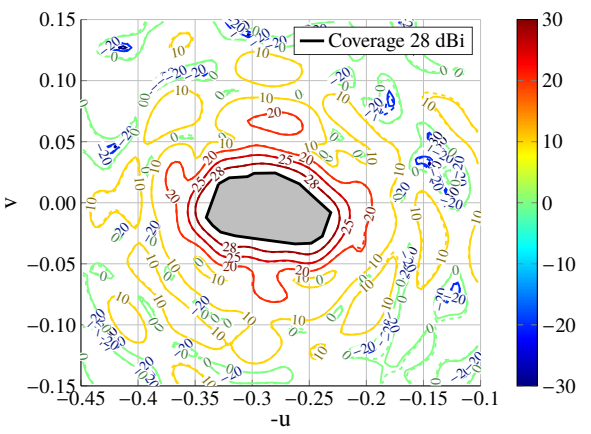

(e)

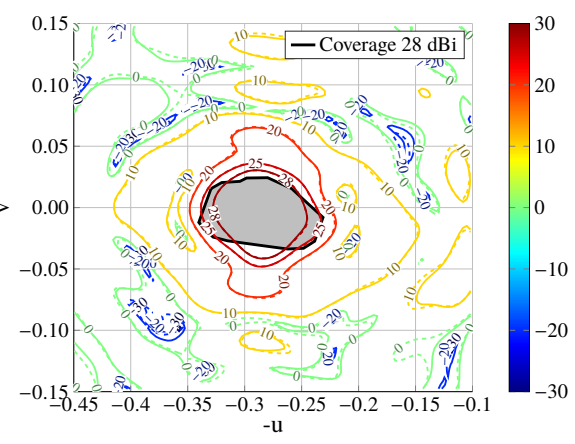

(c)

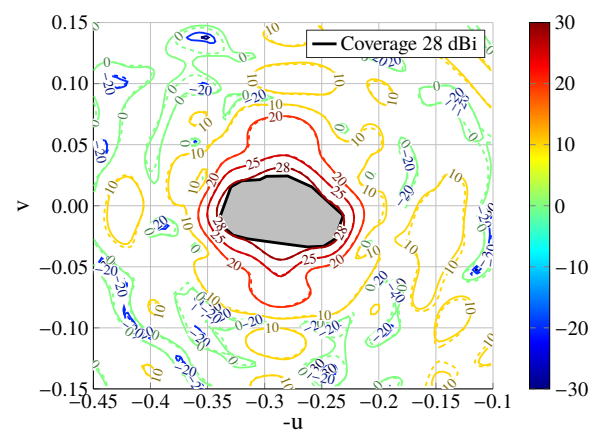

(f)

Fig. 3. Initial (top) and optimized (bottom) radiation patterns for Y polarization (all of them in $\mathrm{dBi}$ ) at (a), (d) 10.95 GHz; (b), (e) 11.85 GHz; and (c), (f) $12.75 \mathrm{GHz}$ simulated with MoM-LP (solid lines) and SVR (dashed lines). (u,v) coordinates are in the reflectarray coordinate system [1].

two different distributions of the angles of incidence: the real angles at each reflectarray element and the discretized angles of incidence employed by the SVR and given in Section II. As it can be seen, the minimum gain predicted by the SVR is close to the one computed using MoM-LP, and more similar results are obtained when both tools employ the same angles of incidence. This is consistent since the error of the surrogate models is very low, as shown in Section II, and it translates to a good prediction of the radiation patterns.

Regarding the optimized layout, it completely fulfils specifications at $11.40 \mathrm{GHz}, 11.85 \mathrm{GHz}$ and $12.30 \mathrm{GHz}$, and also at $12.75 \mathrm{GHz}$ for polarization Y. At $10.75 \mathrm{GHz}$ it is close to fulfil the $28 \mathrm{dBi}$ requirement. It is noteworthy to remark the improvement at $12.75 \mathrm{GHz}$, since the minimum copolar gain has improved more than $10 \mathrm{~dB}$ and $4 \mathrm{~dB}$ for polarization $\mathrm{X}$ and $\mathrm{Y}$, respectively. In the case of $10.95 \mathrm{GHz}$, the improvement is better than $1.5 \mathrm{~dB}$ for both polarizations. It has been checked that the reflectarray fulfils the $28 \mathrm{dBi}$ in the range $11.05 \mathrm{GHz}-$ $12.50 \mathrm{GHz}$ in dual-linear polarization, which corresponds to a $12.2 \%$ bandwidth. This has been achieved by only employing one degree of freedom per cell and polarization.

Finally, the multi-frequency optimization was carried out in an Intel Xeon E5-2630 v4 CPU at 2.2 GHz. While using the MoM-LP tool each iteration took a mean time of $735.1 \mathrm{~s}$ (more than $12 \mathrm{~min}$ ), using the SVR it was reduced to $34.96 \mathrm{~s}$ per iteration: a speed-up larger than one order of magnitude (speed-up of 21). Taking into account that the optimization took close to 500 iterations, the total time savings were more than $90 \mathrm{~h}$ (from $102 \mathrm{~h}$ using MoM-LP to $4.8 \mathrm{~h}$ using SVR), while keeping a high degree of accuracy by using SVR. The speed-up for the monochromatic case is 3.6, further demonstrating the usefulness of SVRs for wideband optimization.

\section{CONCLUSIONS}

In this letter, support vector machines for regression (SVR) have been employed to perform a multi-frequency design of a very large shaped-beam reflectarray for direct broadcast satellite (DBS) application in dual-linear polarization. The SVR yields surrogate models of the unit cell electromagnetic behaviour to predict the values of the reflection coefficients at different frequencies and angles of incidence. Each SVR training takes less than 40 seconds, while obtaining a high degree of accuracy when compared to MoM-LP. The mean test error for all coefficients for all angles of incidence and frequencies is $-40.9 \mathrm{~dB}$. A layout obtained at central frequency is used as starting point for a multi-frequency optimization considering five equispaced frequencies. After the optimization, the reflectarray completely fulfils the $28 \mathrm{dBi}$ requirements in a $12.2 \%$ bandwidth, and it is close to fulfil requirements in a $15 \%$ bandwidth. This has been achieved using only one degree of freedom per unit cell and polarization. The improvement in minimum copolar gain at $12.75 \mathrm{GHz}$ is more than $10 \mathrm{~dB}$ and $4 \mathrm{~dB}$ for polarizations $\mathrm{X}$ and $\mathrm{Y}$, respectively. Finally, the optimization procedure is accelerated more than one order of magnitude using SVR instead of MoM-LP in the analysis step, saving a considerable amount of time, more than $90 \mathrm{~h}$ in the present case, while obtaining a high degree of accuracy with regard to MoM-LP simulations. This work demonstrates the suitability of SVR analysis-based broadband design of very large reflectarrays for space applications. 


\section{REFERENCES}

[1] J. Huang and J. A. Encinar, Reflectarray Antennas. Hoboken, NJ, USA: John Wiley \& Sons, 2008.

[2] D. M. Pozar, "Bandwidth of reflectarrays," Electron. Lett., vol. 39, no. 21, pp. 1490-1491, Oct. 2003.

[3] J. A. Encinar and J. A. Zornoza, "Three-layer printed reflectarrays for contoured beam space applications," IEEE Trans. Antennas Propag., vol. 52, no. 5, pp. 1138-1148, May 2004.

[4] J. H. Yoon, Y. J. Yoon, W.-S. Lee, and J.-H. So, "Broadband microstrip reflectarray with five parallel dipole elements," IEEE Antennas Wireless Propag. Lett., vol. 14, pp. 1109-1112, 2015.

[5] L. Moustafa, R. Gillard, F. Peris, R. Loison, H. Legay, and E. Girard, "The phoenix cell: A new reflectarray cell with large bandwidth and rebirth capabilities," IEEE Antennas Wireless Propag. Lett., vol. 10, pp. 71-74, 2011.

[6] D. M. Pozar, "Wideband reflectarrays using artificial impedance surfaces," Electron. Lett., vol. 43, no. 3, pp. 148-149, Feb. 2007.

[7] L. Guo, P.-K. Tan, and T.-H. Chio, "On the use of single-layered subwavelength rectangular patch elements for broadband folded reflectarrays," IEEE Antennas Wireless Propag. Lett., vol. 16, pp. 424-427, 2017.

[8] P.-Y. Qin, Y. J. Guo, and A. R. Weily, "Broadband reflectarray antenna using subwavelength elements based on double square meander-line rings," IEEE Trans. Antennas Propag., vol. 64, no. 1, pp. 378-383, Jan. 2016.

[9] J. A. Encinar, M. Arrebola, M. Dejus, and C. Jouve, "Design of a 1metre reflectarray for DBS application with $15 \%$ bandwidth," in First European Conference on Antennas and Propagation (EuCAP), Nice, France, Nov. 6-10, 2006, pp. 1-5.

[10] E. Carrasco, J. A. Encinar, and M. Barba, "Bandwidth improvement in large reflectarrays by using true-time delay," IEEE Trans. Antennas Propag., vol. 56, no. 8, pp. 2496-2503, Aug. 2008.

[11] J. A. Encinar, M. Arrebola, and G. Toso, "A parabolic reflectarray for a bandwidth improved contoured beam coverage," in The Second European Conference on Antennas and Propagation (EuCAP), Edinburgh, Scotland, United Kingdom, Nov. 11-16 2007, pp. 1-5.

[12] M. Zhou, S. B. Sørensen, O. Borries, and E. Jørgensen, "Analysis and optimization of a curved transmit-receive contoured beam reflectarray," in The $9^{\text {th }}$ European Conference on Antennas and Propagation (EuCAP), Lisbon, Portugal, Apr. 13-17, 2015, pp. 1-5.

[13] H. Legay, D. Bresciani, E. Labiole, R. Chiniard, and R. Gillard, "A multi facets composite panel reflectarray antenna for a space contoured beam antenna in Ku band," Progr. Electromagn. Res. B, vol. 54, pp. 1-26, Aug. 2013.

[14] M. Zhou, S. B. Sørensen, P. Meincke, and E. Jørgensen, "Design and optimization of multi-faceted reflectarrays for satellite applications," in The $8^{\text {th }}$ European Conference on Antennas and Propagation (EUCAP), The Hague, The Netherlands, Apr. 6-11, 2014, pp. 1423-1427.

[15] C. T. Tong, C. E. F. Raelene, and K. C. Tian, "Comparison of simulated performance of faceted \& flat reflectarray antennas," in International Symposium on Antennas and Propagation (ISAP), Busan, South Korea, Oct. 23-26, 2018, pp. 1-2.

[16] A. Massa, G. Oliveri, M. Salucci, N. Anselmi, and P. Rocca, "Learningby-examples techniques as applied to electromagnetics," J. Electromagn. Waves Appl., vol. 32, no. 4, pp. 516-541, 2018.

[17] P. Robustillo, J. Zapata, J. A. Encinar, and J. Rubio, "ANN characterization of multi-layer reflectarray elements for contoured-beam space antennas in the Ku-band," IEEE Trans. Antennas Propag., vol. 60, no. 7. pp. 3205-3214, Jul. 2012

[18] A. Freni, M. Mussetta, and P. Pirinoli, "Neural network characterization of reflectarray antennas," Int. J. Antennas Propag., vol. 2012, pp. 1-10, May 2012.

[19] F. Güneş, S. Nesil, and S. Demirel, "Design and analysis of Minkowski reflectarray antenna using 3-D CST Microwave Studio-based neural network model with particle swarm optimization," Int. J. RF Microw. Comput. Eng., vol. 23, no. 2, pp. 272-284, Mar. 2013.

[20] P. Robustillo, J. Zapata, J. A. Encinar, and M. Arrebola, "Design of a contoured-beam reflectarray for a eutelsat european coverage using a stacked-patch element characterized by an artificial neural network," IEEE Antennas Wireless Propag. Lett., vol. 11, pp. 977-980, 2012.

[21] T. Shan, M. Li, S. Xu, and F. Yang, "Synthesis of refiectarray based on deep learning technique," in Cross Strait Quad-Regional Radio Science and Wireless Technology Conference (CSQRWC), Xuzhou, China, Jul. 21-24, 2018, pp. 1-2.
[22] M. Salucci, L. Tenuti, G. Oliveri, and A. Massa, "Efficient prediction of the EM response of reflectarray antenna elements by an advanced statistical learning method," IEEE Trans. Antennas Propag., vol. 66, no. 8, pp. 3995-4007, Aug. 2018.

[23] D. R. Prado, J. A. López-Fernández, G. Barquero, M. Arrebola, and F. Las-Heras, "Fast and accurate modeling of dual-polarized reflectarray unit cells using support vector machines," IEEE Trans. Antennas Propag., vol. 66, no. 3, pp. 1258-1270, Mar. 2018.

[24] D. R. Prado, J. A. López-Fernández, M. Arrebola, and G. Goussetis, "Support vector regression to accelerate design and crosspolar optimization of shaped-beam reflectarray antennas for space applications," IEEE Trans. Antennas Propag., vol. 67, pp. 1659-1668, Mar. 2019.

[25] S. V. Hum and B. Du, "Equivalent circuit modeling for reflectarrays using Floquet modal expansion,” IEEE Trans. Antennas Propag., vol. 65, no. 3, pp. 1131-1140, Mar. 2017.

[26] R. Florencio, R. R. Boix, and J. A. Encinar, "Enhanced MoM analysis of the scattering by periodic strip gratings in multilayered substrates," IEEE Trans. Antennas Propag., vol. 61, no. 10, pp. 5088-5099, Oct. 2013.

[27] J. A. Zornoza and J. A. Encinar, "Efficient phase-only synthesis of contoured-beam patterns for very large reflectarrays," Int. J. RF Microw. Comput. Eng., vol. 14, no. 5, pp. 415-423, Sep. 2004.

[28] M. Arrebola, J. A. Encinar, and M. Barba, "Multifed printed reflectarray with three simultaneous shaped beams for LMDS central station antenna," IEEE Trans. Antennas Propag., vol. 56, no. 6, pp. 1518-1527, Jun. 2008.

[29] D. R. Prado, J. Álvarez, M. Arrebola, M. R. Pino, R. G. Ayestarán, and F. Las-Heras, "Efficient, accurate and scalable reflectarray phase-only synthesis based on the Levenberg-Marquardt algorithm," Appl. Comp. Electro. Society Journal, vol. 30, no. 12, pp. 1246-1255, Dec. 2015.

[30] R. Florencio, J. A. Encinar, R. R. Boix, V. Losada, and G. Toso, "Reflectarray antennas for dual polarization and broadband telecom satellite applications," IEEE Trans. Antennas Propag., vol. 63, no. 4, pp. 1234-1246, Apr. 2015

[31] J. A. Encinar, R. Florencio, M. Arrebola, M. A. Salas-Natera, M. Barba, J. E. Page, R. R. Boix, and G. Toso, "Dual-polarization reflectarray in Ku-band based on two layers of dipole arrays for a transmit-receive satellite antenna with South American coverage," Int. J. Microw. Wirel. Technol., vol. 10, no. 2, pp. 149-159, 2018.

[32] C.-C. Chang and C.-J. Lin, "LIBSVM: A library for support vector machines," ACM Trans. Intell. Syst. Technol., vol. 2, no. 3, pp. 27:127:27, Apr. 2011, software available at https://www.csie.ntu.edu.tw/ $\sim$ cjlin/libsvm.

[33] B. Schölkopf and A. J. Smola, Learning with Kernels, 1st ed. Cambridge, Massachusetts: The MIT Press, 2001.

[34] O. M. Bucci, G. D'Elia, G. Mazzarella, and G. Panariello, "Antenna pattern synthesis: a new general approach," Proc. IEEE, vol. 82, no. 3 , pp. 358-371, Mar. 1994.

[35] D. R. Prado, J. A. López-Fernández, M. Arrebola, M. R. Pino, and G. Goussetis, "General framework for the efficient optimization of reflectarray antennas for contoured beam space applications," IEEE Access, vol. 6, pp. 72 295-72 310, 2018.

[36] D. R. Prado, M. Arrebola, M. R. Pino, R. Florencio, R. R. Boix, J. A. Encinar, and F. Las-Heras, "Efficient crosspolar optimization of shaped-beam dual-polarized reflectarrays using full-wave analysis for the antenna element characterization," IEEE Trans. Antennas Propag., vol. 65, no. 2, pp. 623-635, Feb. 2017. 